\title{
Measuring Thickness and Pretilt in Reflective Vertically Aligned Nematic Liquid Crystal Displays
}

\author{
B. Cerrolaza, M. A. Geday, J. M. Otón, X. Quintana, \\ and N. Bennis \\ Department Tecnología Fotónica, ETSI Telecomunicación, Universidad \\ Politécnica de Madrid, Ciudad Universitaria, Madrid, Spain
}

\begin{abstract}
Pretilt angle is a parameter of the utmost importance in the ultimate performance of vertically-aligned negative nematic LC displays. When these devices work in reflective mode, as is the LCOS microdisplays, accurate measurement of pretilt angles becomes a difficult problem, since usual experimental setups based on retardation of the polarization components of the impinging light are proportional to the product effective birefringence $\left(n_{\text {eff }}-n_{o}\right)$ times thickness, and any attempt to separate these variables is cancelled out by symmetry. This work shows a relatively simple method capable of separating both variables. An experimental setup specifically aimed at vertically aligned reflective cells has been prepared. At the same time, a simulation model has been developed taking into account the properties of actual reflective displays. Comparison between experimental and theoretical results shows some discrepancies that can be explained assuming that the LC profile contains a residual twist. Including that twist in the model, an excellent agreement between theory and experiment has been achieved. Matching of simulations and measurements yields to the separate determination of pretilt angle and thickness and gives good estimates for the residual twist angle.
\end{abstract}

Keywords: pretilt angle; residual twist; vertically aligned nematic

\section{INTRODUCTION}

Vertically aligned nematic (VAN) liquid crystal displays having negative dielectric anisotropy are becoming popular in direct-view display applications VANs are also one of the most common choices for high-end projection microdisplays in projection devices where VANs are usually driven by an active matrix silicon backplane (liquid crystal on silicon, LCOS), the device working in reflective mode. 
VAN materials are characterized by a negative dielectric anisotropy, which means that the LC molecules will tend to orient perpendicularly to any applied electric field. If the alignment were strictly vertical, the direction of the $\mathrm{LC}$ reorientation would be undetermined since any position within the plane parallel to the outer plates would be energetically equivalent. Moreover, the response time would be jeopardized because the electric torque is null for pure vertical alignment. These problems can be avoided by inducing a certain pretilt in the vertical orientation thus favoring a specific switching plane and speeding up the switching time. However, pretilt has also a drawback: the relaxed state becomes birefringent, and the contrast decays dramatically as pretilt increases. Therefore, choosing a precise pretilt is an important tradeoff when designing real LCOS VAN devices. It follows that accurate measurement of pretilt in actual working devices and their spatial homogeneity across the cell - is extremely important.

Several techniques have been proposed and widely applied to measure the pretilt angle of LC cells, such as the magnetic null method, the crystal rotation technique, the polarizer rotation method, and the phase retardation measurement method. All these methods are accurate and reliable for the pretilt angle measurement of transmissive cells.

The problem in measuring the pretilt angle in reflective VAN cells is that the birefringence that can be measured at normal incidence is proportional both to the effective birefringence $\left(n_{\text {eff }}-n_{o}\right)$ and to the cell thickness. The methods proposed for measuring pretilt angles in reflective cells suffer from problems in measuring the dark current of the detector and the normalization of the incident light.

In this work we describe a procedure capable of separating the thickness and pretilt dependences. The procedure is based in sample rotation while acquiring the light intensity at normal incidence and at oblique incidence.

\section{THEORY}

The mathematical model presented here is based in the Jones matrix theory, and has been developed in Maple 10. The model includes the effects of interfaces and oblique incidence in the impinging beam intensity. The cell is rotating about the point where light is impinging, and the incidence angle may vary from normal incidence to any angle $\gamma$ from the normal axis. 
The coordinate system and angles employed in the model are shown in Figure 1. All referenced angles $(\beta, \gamma, \theta, \varphi)$ are internal, e.g., $\gamma$ is the angle of the incident light after crossing the glass-LC interface. The cell plates are onto the XY plane and $\mathrm{Z}$ is the normal to the surface. The pretilt angle $\beta$ is the angle that is being measured. Rotation of the cell about the $\mathrm{Z}$ axis is defined by $\varphi$, while $\theta$ is the difference between $\gamma$ and $\beta \cdot \vec{N}$ is the director vector, forming an angle $\beta$ already defined with normal $\mathrm{Z} \cdot \vec{K}$ is the propagation vector of the incident beam forming an angle $\gamma$ already defined with normal Z.

Birefringence, as "seen" by the incident light, is not the difference between extraordinary and ordinary refractive indices $\left(\Delta_{n}=\left|n_{e}-n_{o}\right|\right)$ but the difference between the ordinary index and an effective index $n_{\text {eff }}$ defined as:

$$
n_{e f f}=\frac{1}{\sqrt{\frac{\cos \beta^{2}}{n_{e}^{2}}+\frac{\sin \beta^{2}}{n_{o}^{2}}}}
$$

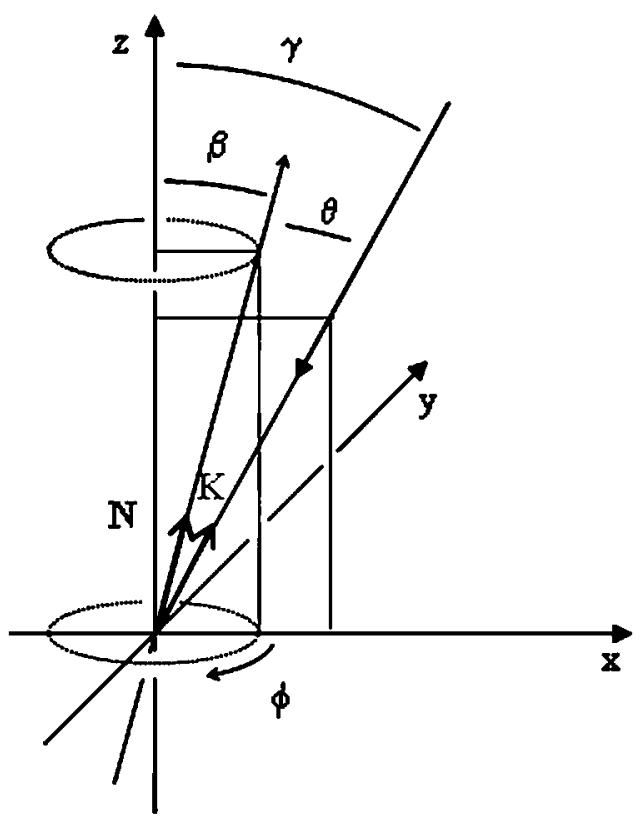

FIGURE 1 Coordinate system and notation angles employed in the mathematical model. 
When light propagates across the LC, a linear retardation $\delta$ occurs:

$$
\delta=\frac{2 \pi \cdot \Delta_{n} \cdot L}{\lambda}
$$

where $\lambda$ is the light wavelength, $L$ is the cell thickness, and $\Delta_{n}$ is the birefringence experienced by the incoming light (actually $\Delta_{n}$ should be labelled $\Delta_{n e f f}$, since it always refers to effective index $n_{\text {eff }}$ we use $\Delta_{n}$ for simplicity). The light path $L_{\text {eff }}$ inside the cell depends on the incidence angle $\gamma$ :

$$
L_{e f f}=\frac{L}{\cos \gamma}
$$

After unsuccessful trials of matching experimental results within this framework, a small circular retardation has been included as well. This is named hereinafter as "residual twist," although its actual origin is still under study. It is defined by means of another angle $\rho$.

A Jones matrix is defined taking into account both, linear and circular retardations

$$
M=\left[\begin{array}{cc}
\cos w_{i}+\frac{\frac{1}{\frac{1}{2} j \delta_{i}-\sin w_{i}}}{w_{i}} & -\rho \cdot \frac{\sin w_{i}}{w_{i}} \\
\rho \cdot \frac{\sin w_{i}}{w_{i}} & \cos w_{i}-\frac{\frac{1}{2} j \delta_{i}-\sin w_{i}}{w_{i}}
\end{array}\right]
$$

where $w_{i}=\sqrt{\rho^{2}+\left(\delta_{i} / 2\right)^{2}}$, making $i=1$ for the incoming light path and $i=2$ for the outcoming light path after reflection in the backplane mirror. This index notation is kept along the whole mathematical development.

The Jones matrix of the backplane aluminum mirror of the LCOS device is

$$
\text { Mirr }=\left[\begin{array}{cc}
-1 & 0 \\
0 & 1
\end{array}\right]
$$

A second coordinate system is required to simplify the expressions. The system is defined by the impinging beam direction, taken as $\mathrm{Z}^{\prime}$ axis, and its normal plane $\mathrm{X}^{\prime} \mathrm{Y}^{\prime}$. Let $\alpha$ be the angle between the projection of the molecular director $\bar{N}$ on the $\mathrm{X}^{\prime} \mathrm{Y}^{\prime}$ plane and the axis $\mathrm{X}^{\prime}$.

With these definitions, the light intensity of the outcoming beam after its double path through the LC and the mirror reflection is:

$$
I_{\text {out }}=J_{\text {out }}[1] \cdot J_{\text {out }}^{*}[1]
$$

where $I_{\text {out }}$ is the polarized light measured in the photodetector at the output after having incided with polarizaed light in the cell: 


$$
\begin{aligned}
J_{\text {out }}= & {\left[\begin{array}{cc}
\cos \alpha_{2} & \sin \alpha_{2} \\
-\sin \alpha_{2} & \cos \alpha_{2}
\end{array}\right] \cdot\left[\begin{array}{cc}
\cos w_{2}+\frac{\frac{1}{2} j \delta_{2} \cdot \sin w_{2}}{w_{2}} & -\rho \cdot \frac{\sin w_{2}}{w_{2}} \\
\rho \cdot \frac{\sin w_{2}}{w_{2}} & \cos w_{2}-\frac{\frac{1}{2} j \delta_{2} \cdot \sin w_{2}}{w_{2}}
\end{array}\right] } \\
& \cdot\left[\begin{array}{cc}
\cos \alpha_{2} & -\sin \alpha_{2} \\
\sin \alpha_{2} & \cos \alpha_{2}
\end{array}\right] \cdot\left[\begin{array}{cc}
-1 & 0 \\
0 & 1
\end{array}\right] \cdot\left[\begin{array}{cc}
\cos \alpha_{1} & -\sin \alpha_{1} \\
\sin \alpha_{2} & \cos \alpha_{1}
\end{array}\right] \\
& \cdot\left[\begin{array}{cc}
\cos w_{1}+\frac{\frac{1}{2} j \delta_{1} \cdot \sin w_{1}}{w_{1}} & -\rho \cdot \frac{\sin w_{1}}{w_{1}} \\
\rho \cdot \frac{\sin w_{1}}{w_{1}} & \cos w_{1}-\frac{\frac{1}{2} j \delta_{1} \cdot \sin w_{1}}{w_{1}}
\end{array}\right] \cdot\left[\begin{array}{cc}
\cos \alpha_{1} & \sin \alpha_{1} \\
-\sin \alpha_{1} & \cos \alpha_{1}
\end{array}\right] \cdot\left[\begin{array}{l}
0 \\
1
\end{array}\right]
\end{aligned}
$$

This mathematical model describes the intensity curves at the output of a reflective cell when light impinges at a certain (internal) angle $\gamma$. Normal incidence $(\gamma=0)$, is just a particular case. Oblique incidence contains more information since the characteristic symmetry of normal incidence is avoided. Comparison with experimental results will be done by fixing all variables except the ration angle, and

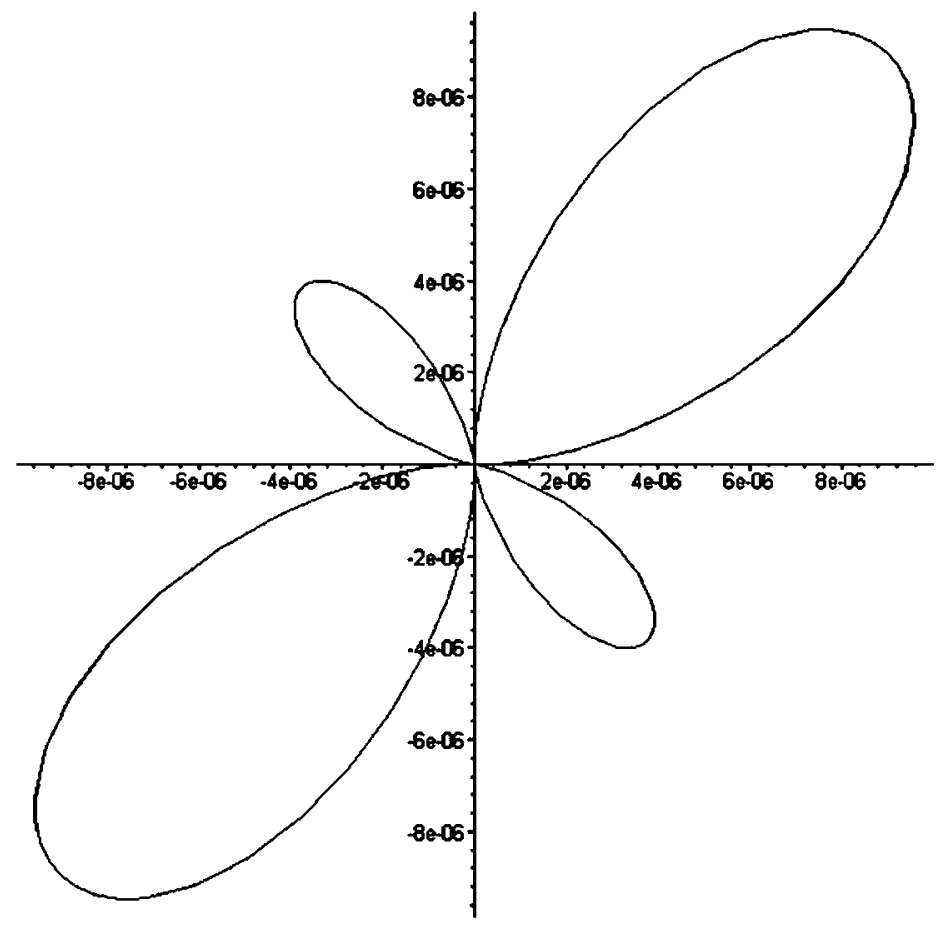

FIGURE 2 Simulation intensity curve for a VAN cell with $\mathrm{L}=2 \mu \mathrm{m}, \beta=5^{\circ}$, $\gamma=10^{\circ}, \rho=2^{\circ}$ and $\Delta_{\mathrm{n}}=0.15$. 
plotting light intensity for all possible rotations $\phi=[0,2 \pi]$. Moreover, the simulation predicts the range of incidence angles for which asymmetry of the output intensity is maximized for a given pretilt angle (Fig. 2). This issue is important since uncertainties in experimental results are high due to the weakness of the output intensity signals (we are measuring an unswitched high contrast LCOS VAN device).

\section{EXPERIMENTAL}

A picture and a sketch of the experimental setup are shown in Figure 3 . It includes a He-Ne laser working at $\lambda=543 \mathrm{~nm}$, two beam-splitters, several first-face mirrors, a motor for sample rotation, two polarizing cubes and a photodetector. The setup allows measurements at normal and oblique incidence without modifying any part; data are separately collected from the normal or the oblique light path while the other path is blocked by means of a shutter. Rotation of the sample and data acquisition are controlled by a LabView software specifically designed for this setup.
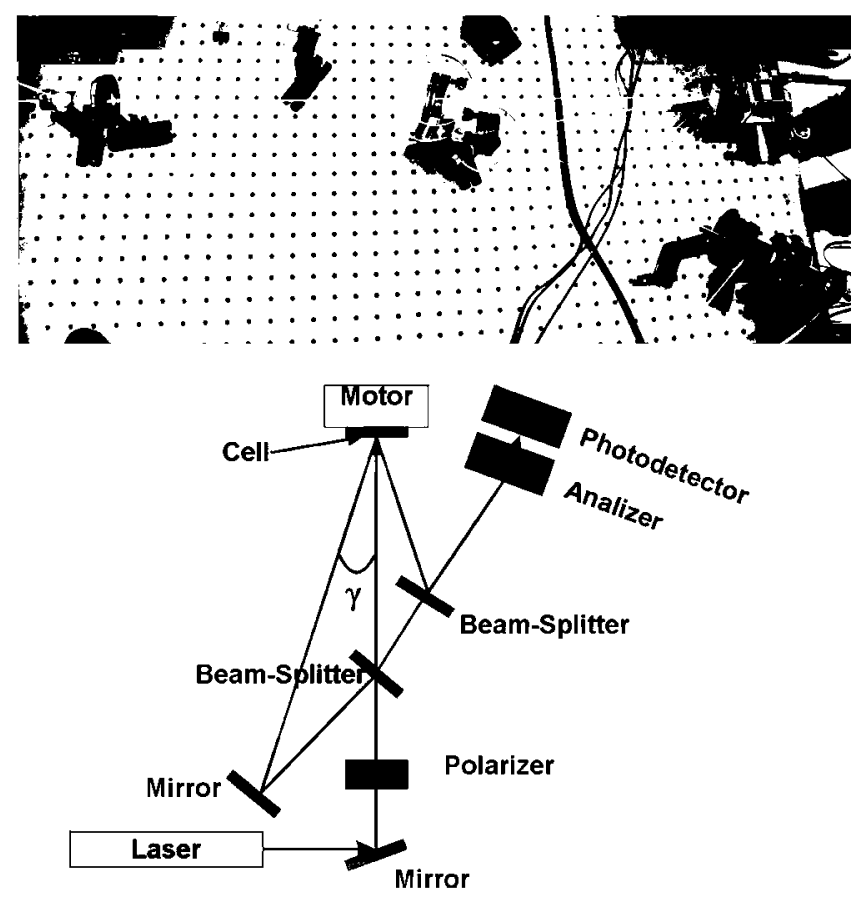

FIGURE 3 A picture and a sketch of the experimental setup used for measurements. 


\section{Measurements at $\gamma=7.51^{\circ}$}

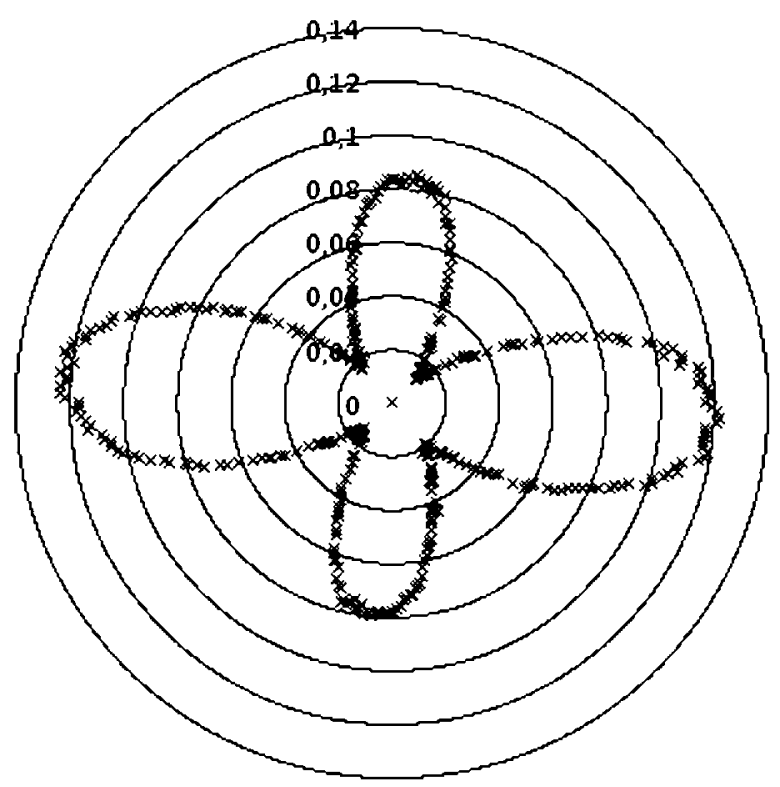

FIGURE 4 Experimental results of a commercial cell obtained with an incidence angle of $7.51^{\circ}$.

The experimental procedure begins with a mirror placed instead of the sample. This is used to minimize the reflected light by rotating the polarizers. Polarizers are considered to be crossed when the minimum transmission is achieved. Then the mirror is substituted by the sample being tested. Oblique and normal transmissions are collected separately using the shutter on either light path while the sample is rotated at $1 \mathrm{~Hz}$ (Fig. 4).

\section{RESULTS}

The collected measurements have been stored in an Excel sheet and adjusted to the theoretical model using the Solver Add-in of the same program. Convergence has been established as a non-linear leastsquares fit whose variables are the cell thickness, the pretilt, and the residual twist. No constraints are imposed to these variables. Several consecutive trials are usually required for the program to converge.

Figure 5 shows a typical result. Once convergence is achieved in the oblique incidence case, the same values are used in the normal 

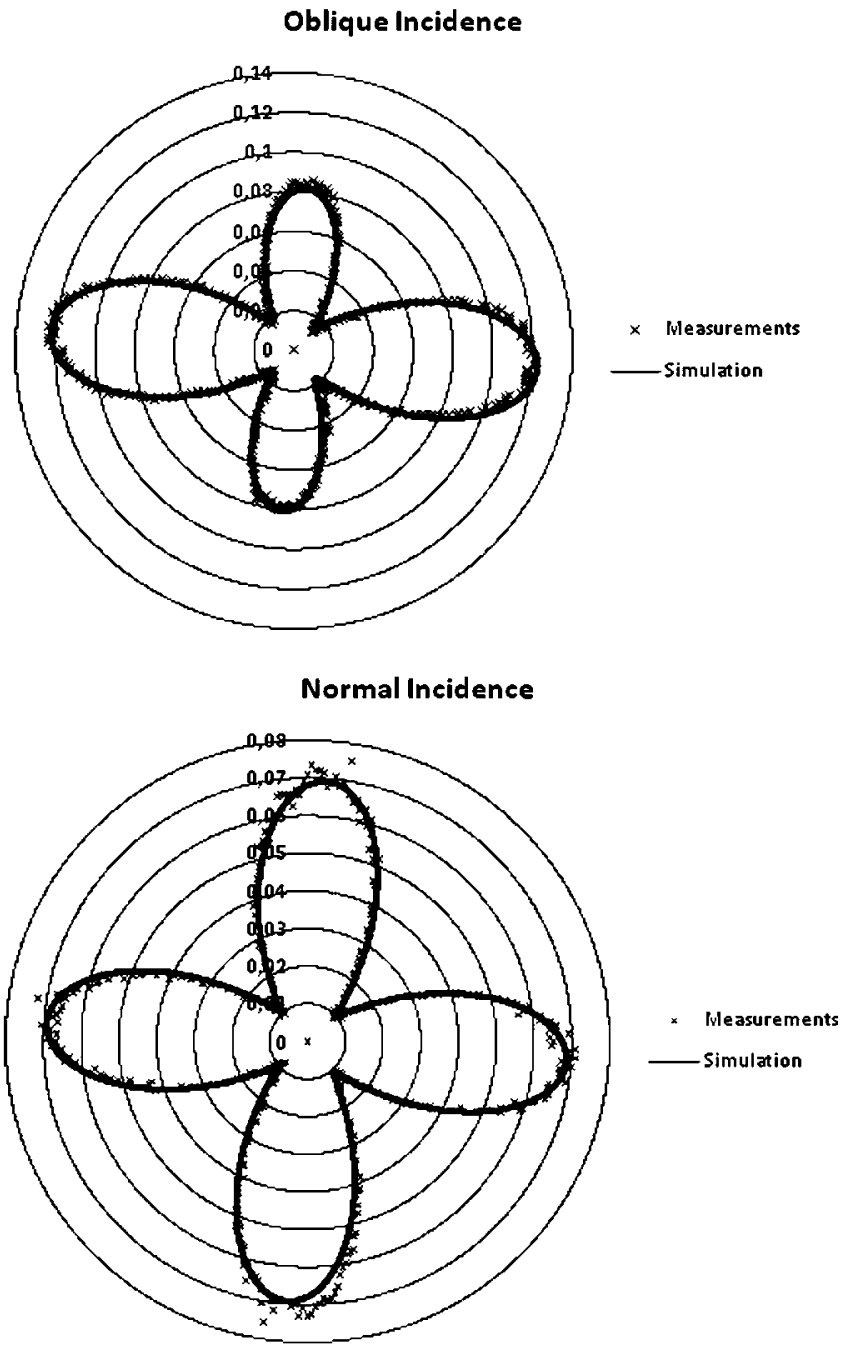

FIGURE 5 Experimental results and theoretical fit for a commercial cell studied.

incidence case. The only variable in the second fit is the light intensity, which cannot be theoretically derived because the beam splitters of the setup introduce uncertainties in the transmission intensity. An accurate matching of the second case using the parameters obtained in the first fit is considered to be a confirmation of the correctness of the whole procedure. 
TABLE 1 The Results Obtained for a Commercial Cell with an Incidence Angle $\gamma=7.51^{\circ}$ and $\lambda=543 \mathrm{~nm}$. The Refractive Indices of the Material are $n_{e}=1.5595, n_{o}=1.4775$

\begin{tabular}{lc}
\hline Parameters & Results \\
\hline Thickness L & $2,4 \mu \mathrm{m}$ \\
Pretilt $\beta$ & $2,841^{\circ}$ \\
Residual twist $\rho$ & $2,258^{\circ}$ \\
\hline
\end{tabular}

The results for the sample of the figure are summarized in (Table 1).

\section{CONCLUSIONS}

An experimental setup supported by a theoretical development has been designed for accurate measurement of thickness, pretilt and residual twist of reflective VAN LC cells. The method is useful to predict optimized design parameters in this kind of microdisplays. Moreover, it can be used as a characterization tool to explore the spatial homogeneity of VAN cells.

The method overcomes the ambiguity derived from the nearly symmetric response of reflective cells under most experimental setups. Measurements are extremely sensible to tiny variations of the analyzed parameters, what ultimately leads to accurate estimations of the same.

\section{REFERENCES}

Lu, R., Hong, Q., Wu, S.-T., Peng, K.-H., \& Hsieh, H.-S. (2006). J. Display Technol., $2,319-326$.

Yu, X. J. \& Kwok, H. S. (2006). Appl. Phys. Lett., 89, 031104.

Takatoh, K., Sakamoto, M., Hasegawa, R., Koden, M., Itoh, N., \& Hasegawa, M. (2005). Alignment Technologies and Applications of Liquid Crystal Devices, Taylor \& Francis Inc: NY.

Tang, S. T. \& Kwok, H. S. (2001). J. Appl. Phys., 89, 80-85.

Han, K. Y., Miyashita T., \& Uchida, T. (1994). Mol. Cryst. Liq. Cryst., 241, 147-157.

Gwag, J. S., Lee, S. H., Park, K, Park, W. S., Han, K., \& Jhun, C. G. (2003). J. Appl. Phys., 8, 4936.

Rho, B. G., Kim, J. S., \& Kim, J. K. (1998). Proc. Asia Display, 98, 245-248.

Kawamura, M., Goto, Y., \& Sato, S. (2003). Proc. SPIE 03, 5213, 250-257.

Cuypers, D., De Smet, H., Van Doorselaer, G., Van Den Steen, J., \& Van Calster, A. (2003). Proc. SPIE 03, 5002, 62.

Li, J., Wen, C., Gauza, S., Lu, R., \& Wu, S. (2005). J. Displ. Tech., 1, 51.

Hwang, S. J., Lin, S. T., \& Lai, C. H. (2006). Opt. Comm., 260, 614-620.

Hsu, J. S., Liang, B. J., \& Chen, S. H. (2005). IEEE Trans Electron. Dev., $52(5), 918$.

Geday, M. A. \& Glazer, A. M. (2002). J. Appl. Cryst., 35(2), 185-190. 